\title{
Pre- and post-transplant risk factors for renal dysfunction in the patients with preserved renal function at 1 month after liver transplantation: a national cohort study using Korean Organ Transplantation Registry (KOTRY)
}

\author{
Deok Gie Kim¹, Jae Geun Lee², Myoung Soo Kim², Dong Jin Joo² \\ ${ }^{1}$ Department of Surgery-Transplantation, Wonju Severance Christian Hospital, Wonju, Korea \\ ${ }^{2}$ Department of Surgery-Transplantation, Yonsei University College of Medicine, Seoul, Korea
}

Background: Renal dysfunction (RD) is an important long-term complication after liver transplantation (LT) which is associated with patient survival. This study investigated pre- and post-transplant risk factors for RD in patient with preserved renal function at 1 month after LT using the Korean Organ Transplantation Registry (KOTRY) data.

Methods: We performed retrospective cohort study using data of recipients who underwent LT between April 2014 and December 2018 and were registered in KOTRY. We defined RD as 40\% decline of eGFR from the value at 1 month and at least below $60 \mathrm{~mL} / \mathrm{min} / 1.73 \mathrm{~m}^{2}$. We performed multivariable Cox regression analyses for pre-transplant and within-one-month risk factors for RD. For post-transplant risk factors, we performed matched analyses according to 8 factors: biopsy-proven acute rejection, infection, bile duct complication, recurrence of HCC, new-onset diabetes mellitus (DM) after transplantation, type of immunosuppressants, steroid withdraw, and type of anti-HBV prophylaxis.

Results: Among 2,274 eligible patients, 251 (11.3\%) developed RD during 36.6 $\$ 14.4$ of mean follow-up period. From multivariable Cox regression analyses, age, female sex, lower body mass index, pre-transplant DM, alcoholic liver disease, above Milan hepatocellular carcinoma (HCC), low Karnofsky performance status score at 1 month, bile duct complication within 1 month and lower estimated glomerular filtration rate at LT were identified as independent risk factors for RD after LT. Among post-transplant factors after 1 month, patients with recurrence of HCC $(P=0.013)$, infection $(P=0.003)$, and tacrolimus-mono treatment (vs. tacrolimus-based dual treatment, $P=0.022$ ) showed higher $R D$ than control patients without those factors.

Conclusions: This study comprehensively indicates pre- and post-transplant risk factors for RD after LT among patients with preserved renal function at 1 month. Clinicians should consider these risk factors for managing patients during long-term follow-up.

Corresponding author: Dong Jin Joo

E-mail: DJJ00@yuhs.ac

(c) The Korean Society for Transplantation

This is an Open Access article distributed under the terms of the Creative Commons Attribution Non-Commercial License (http://creativecommons.org/licenses/by-nc/4.0/) which permits unrestricted non-commercial use, distribution, and reproduction in any medium, provided the original work is properly cited. 